\title{
Validação dos intervalos de referência hematológicos e bioquímicos estabelecidos para cães domiciliados na Amazônia Oriental, Pará, Brasil*
}

\section{Validation of hematological and biochemical reference intervals established for dogs housed in the Eastern Amazon, Pará, Brazil}

\author{
Natália Rodrigues Marques, ${ }^{* *}$ Rita de Cássia Mousinho Ribeiro, ${ }^{* *}$ Heloise Yacã Balieiro Capela, ${ }^{* * *}$ Ednaldo Silva Filho, ${ }^{* * * * *}$ \\ Danielle Valente Braga, ${ }^{* * *}$ Vanessa Joia de Mello, ${ }^{* * *}$ Wesley Lyeverton Correia Ribeiro, ${ }^{* * * * *}$ Maria Vivina Barros Monteiro***
}

\begin{abstract}
Resumo
O objetivo do presente estudo foi validar intervalos de referências (IRs) para hematologia e bioquímica sanguínea de cães domiciliados da Amazônia Oriental e estabelecer novos IRs para os parâmetros não validados. Foram utilizadas amostras de 44 cães adultos clinicamente saudáveis de diferentes raças e sexos. Na validação, foi utilizada a metodologia proposta pelo Clinical and Laboratory Standards Institute (CLSI) e na determinação dos IRs dos parâmetros não validados foi utilizada a metodologia estatística proposta pela American Society for Clinical Veterinary Pathology (ASCPV). Os IRs foram determinados por meio do programa Excel com o suplemento Reference Value Advisor (versão 2.1). O software realiza os cálculos de acordo com as recomendações CLSI, conforme sugerido pelas diretrizes da ASCVP. Um total de 25 parâmetros (13 hematológicos e 12 bioquímicos) foram submetidos ao processo de validação. Desse total, seis (24\%) parâmetros não foram validados (Hemoglobina, CHGM, eosinófilos, linfócitos, albumina e GGT) e para estes foram estabelecidos novos intervalos. Para a maioria dos parâmetros hematológicos e bioquímicos (76\%), os IRs estabelecidos previamente na literatura ainda são válidos para utilização em cães criados na Amazônia Oriental. Para os parâmetros não validados, apenas para eosinófilos e linfócitos recomendamos que outros trabalhos, com um número maior de animais, sejam realizados para confirmar os nossos resultados ou estabelecer novos IRs. Os IRs estabelecidos para hemoglobina, CHGM, albumina e GGT podem ser utilizados em substituição aos intervalos antigos.
\end{abstract}

Palavras-chave: cão, parâmetros bioquímicos, parâmetros hematológicos.

\begin{abstract}
The aim of the present study was to validate reference intervals (RIs) for hematology and blood biochemistry of dogs domiciled in the Eastern Amazon and to establish new RIs for parameters not yet validated. Samples from 44 clinically healthy adult dogs of different breeds and sexes were used. The methodology proposed by the Clinical and Laboratory Standards Institute (CLSI) was used for validation, and the statistical methodology proposed by the American Society for Clinical Veterinary Pathology (ASCPV) was used to determine the RIs of the non-validated parameters. The IRs were determined using the Excel program with the Reference Value Advisor add-in (version 2.1). The software performs calculations according to CLSI recommendations as suggested by the ASCVP guidelines. A total of 25 parameters (13 hematological and 12 biochemical) were submitted to the validation process. Of this total, six (24\%) parameters were not validated (Hemoglobin, CHGM, eosinophils, lymphocytes, albumin and GGT) and new intervals were established for these. For most hematological and biochemical parameters (76\%), the RIs previously established in the literature are still valid for use in dogs raised in the Eastern Amazon. For non-validated parameters, just for eosinophils and lymphocytes, we recommend that other studies, with a larger number of animals, be carried out to confirm our results or establish new IRs. The established IRs for hemoglobin, CHGM, albumin and GGT can be used in place of the old ranges.
\end{abstract}

Keywords: dog, hematological parameters, biochemical parameters.

${ }^{*}$ Recebido em 20 de junho de 2021 e aceito em 17 de dezembro de 2021.

**Programa de Pós-Graduação em Análises Clínicas, Instituto de Ciências Biológicas (ICB), Universidade Federal do Pará (UFPA), Belém, Pará. Brasil.

Autor para correspondência: mnataliafa@gmail.com.

***Universidade Federal do Pará (UFPA), Instituto de Ciências Biológicas (ICB), Belém, Pará. Brasil.

****Faculdade de Medicina Veterinária, Universidade Federal do Pará (UFPA), Castanhal, Pará. Brasil.

*****Universidade Federal Rural da Amazônia (UFRA), Belém, Pará, Brasil.

${ }^{\star \star \star \star \star \star}$ Departamento de Fisiologia e Farmacologia, Universidade Federal do Ceará (UFC), Fortaleza, Ceará, Brasil. 


\section{Introdução}

Exames hematológicos e bioquímicos auxiliam no diagnóstico e prognóstico de várias doenças veterinárias (RAHMAN, 2018). Entretanto, é indispensável que os intervalos de referência (IRs) estabelecidos para cada espécie sejam comparados aos resultados dos pacientes (BIRGEL JÚNIOR et al., 2001, RUSSELL e ROUSSEL, 2007). O intervalo de referência é um recurso de fundamental importância no parecer médico e para tomada de decisão na avaliação do paciente (GEORGE et al., 2010).

Os IRs são determinados a partir de animais saudáveis, utilizando metodologias padronizadas, cálculos estatísticos e representam uma estimativa dentro da qual $95 \%$ dos indivíduos clinicamente sadios devem ser encontrados (GEORGE et al., 2010). Fatores ambientais, raça, idade, gênero, manejo, nutrição, e estado fisiológico são causas de variações nos IRs para espécies animais.

No Brasil, os IRs para exames laboratoriais usados na medicina são, na maioria dos casos, baseados na bibliografia internacional, porém esses intervalos podem divergir em muitos pontos de cada realidade local (FERREIRA e ANDRIOLO 2008). Na veterinária essa divergência também ocorre, sendo importante estabelecer IRs que se aproximem da realidade local ou, preferencialmente, regional. Segundo Russell e Roussel (2007), há uma maior credibilidade quando um intervalo de referência é definido a partir de amostras originadas da população da qual o paciente faz parte.

O Clinical and Laboratory Standards Institute (CLSI), possui uma norma padrão para validação de IRs. A metodologia proposta pelo CLSI já foi utilizada para cães (NIELSEN et al., 2010). A American Society for Clinical Veterinary Pathology (ASCVP) também disponibiliza recomendações para orientar a determinação e validação de IRs para espécies animais (FRIEDRICHS et al., 2012). O regulamento proposto pela ASCVP foi utilizado recentemente para cães de raças variadas (CHUNG et al., 2016).

No Brasil, os IRs hematológicos e bioquímicos utilizados rotineiramente para cães foram estabelecidos há bastante tempo e, provavelmente, não retratam a realidade atual. Assim, o objetivo do presente estudo foi validar IRs para hematologia e bioquímica sanguinea de cães domiciliados da Amazônia Oriental, Pará, Brasil, e estabelecer, para os parâmetros não validados, novos IRs.

\section{Material e métodos}

O presente estudo foi realizado no Laboratório de Análises Clínicas Divisão Veterinária (LAC-Vet) do Instituto de Ciências Biológicas da Universidade Federal do Pará, Belém, Brasil. O projeto foi previamente aprovado pelo Comitê de Ética para o Uso de Animais da Universidade Federal do Pará (CEUA/UFPA) - protocolo $n^{\circ} 1368140219$. Todos os tutores dos animais foram esclarecidos sobre os objetivos do projeto e assinaram um termo de consentimento conforme exigido pela Resolução Normativa do Conselho Nacional de Controle de Experimentação Animal (CONCEA) N 22, de 25 de junho de 2015 (CONCEA, 2015).

Para compor a população de referência, foram utilizados 44 animais adultos, com idade superior a um ano, de diferentes raças e sexos, que não apresentavam sinais de doença nas duas semanas anteriores ou seguintes à coleta de amostras. Na pesquisa sobre o histórico clínico dos animais, os tutores responderam a um questionário com perguntas sobre alimentação, intervenções cirúrgicas recentes, histórico de doenças, uso de medicações, esquemas completos de vacinação e vermifugação.

Foram excluídos do estudo animais gestantes ou lactantes, com doença clínica em curso, com lesões aparentes, ou fazendo uso de medicações como hormônios ou promotores de crescimento, indutores enzimáticos (corticosteróides ou antiepilepticos), entre outras drogas que pudessem interferir nos exames hematológicos e bioquímicos de acordo com o estabelecido por Friedrichs et al. (2012).

$\mathrm{Na}$ coleta de sangue, os animais foram contidos fisicamente e, quando necessário, foram utilizadas focinheiras. O sangue foi coletado preferencialmente por punção da veia cefálica, utilizando seringas e agulhas descartáveis.

$\mathrm{Na}$ realização dos hemogramas foram coletadas amostras de sangue $(3 \mathrm{~mL})$ em tubos contendo o anticoagulante ácido etilenodiaminotetracético (EDTA). Após as coletas todas as amostras foram refrigeradas e imediatamente remetidas ao LACVet. Todos os animais estavam em jejum de 8 horas antes da coleta.

$\mathrm{Na}$ realização das dosagens bioquímicas, amostras de $3 \mathrm{ml}$ de sangue foram coletadas em tubos sem anticoagulante. As amostras foram imediatamente remetidas ao laboratório e centrifugadas a $3.000 \mathrm{rpm}$ por $10 \mathrm{~min}$.

O sangue total foi utilizado para a determinação do hematócrito, dosagem de hemoglobina, contagem global de hemácias, índices hematimétricos (volume globular médio - VGM, hemoglobina globular média - HGM e concentração de hemoglobina corpuscular média - CHGM), contagem global de plaquetas e contagem global de leucócitos. Foi utilizado um equipamento automatizado Humancount 30 (Human $\mathrm{GmbH}$, Wiesbaden, Germany) calibrado para espécie canina.

A contagem diferencial de leucócitos foi realizada em esfregaços sanguíneos confeccionados imediatamente após a punção da veia cefálica no ambulatório e, posteriormente, corados com Leishman (Laborclin, Pinhais, Brasil) no LAC-Vet. Foram contados 100 leucócitos, os quais foram diferenciados em neutrófilos (bastões e segmentados), eosinófilos, basófilos, monócitos e linfócitos. Os resultados obtidos foram expressos em valores absolutos (células/ $\mu \mathrm{L}$ de sangue).

$\mathrm{Na}$ realização das dosagens bioquímicas, as amostras de soro foram congeladas a temperatura de $-20^{\circ} \mathrm{C}$ até realização das análises (média de 15 dias após a coleta). Procedeu-se a dosagem de colesterol, triglicerídeos, glicose, uréia, creatinina, albumina, globulinas e proteínas totais. Foi determinada a atividade das enzimas alanina aminotransferase (ALT), aspartato aminotransferase (AST), fosfatase alcalina (FA) e gama glutamiltransferase (GGT). As dosagens bioquímicas foram realizadas empregando Kits comerciais (Interteck Katal. Campo Belo, Brasil) em equipamento automatizado CM 200 (Wiener Lab Group. São Paulo, Brasil) utilizando as seguintes metodologias: Colorimétrico para Albumina; método cinético UV para ALT, AST, fosfatase alcalina e GGT; método enzimático colorimétrico para colesterol, creatinina, glicose, proteínas totais, triglicerídeos e uréia e método do cálculo (proteína total - albumina) para 
globulinas. Amostras hemolisadas, lipêmicas ou ictéricas não foram processadas.

Foram utilizados para validação os intervalos estabelecidos por Jain (1993) e Kaneko (1997) para o hemograma e os exames bioquímicos, respectivamente. O procedimento de validação seguiu o protocolo do CLSI (NIELSEN et al., 2010). Para os parâmetros não validados foram estabelecidos novos intervalos utilizando a metodologia estatística proposta para uso em grupos com poucos indivíduos (GEFFRÉ et al., 2011).

Foram selecionados, aleatoriamente, 20 animais clinicamente saudáveis e suas amostras foram usadas para validação de cada parâmetro hematológico ou bioquímico ( $1^{\mathrm{a}}$ amostragem). O parâmetro foi considerado validado quando apenas duas amostras estavam fora do intervalo de referência estabelecido, ou seja, $10 \%$ do total. Quando cinco ou mais amostras estivessem fora do intervalo de referência este era imediatamente rejeitado. Caso o número de amostras divergentes fosse de três a quatro, uma nova amostragem foi realizada ( $2^{a}$ amostragem), utilizando outros 20 animais saudáveis e suas amostras foram submetidas ao mesmo procedimento aplicado (Figura 1). Quando, após a segunda amostragem, os resultados permaneceram rejeitados um novo intervalo de referência foi calculado para cada parâmetro.

Figura 1: Procedimento de validação de intervalos de referência, adaptado de Nielsen et al., (2010).

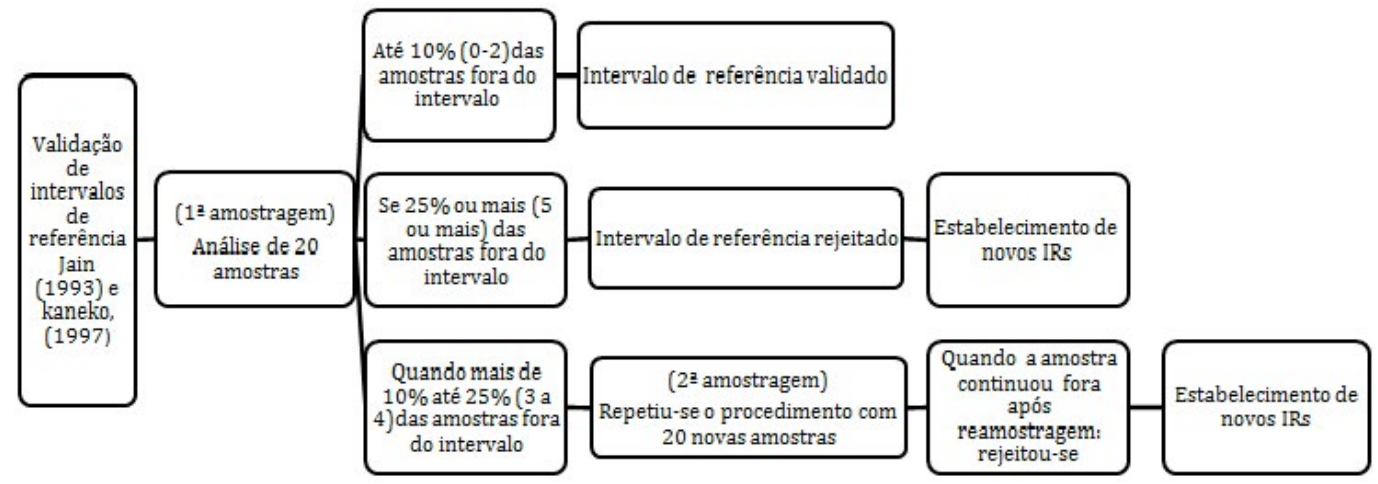

Os IRs foram determinados usando o programa Excel com o suplemento Reference Value Advisor (versão 2.1). O software executa os cálculos de acordo com as recomendações do CLSI, conforme sugerido pelas diretrizes da ASCVP. Os IRs foram calculados usando um método não paramétrico que também calculou o intervalo de confiança (IC) de $90 \%$, exceto quando o número de observações fosse menor de 40 . Nesse caso, foi utilizado o método robusto com os dados transformados em Box - Cox, conforme recomendado pelo software. Estatística descritiva, testes de normalidade de acordo com o método de Anderson - Darling com histogramas e $Q$ - $Q$ gráficos e transformação Box-Cox foram calculados. Para encontrar e remover outliers, foram utilizados os testes de Dixon - Reed e Tukey (RUGGERONE et al., 2018).

\section{Resultados}

Dos 44 cães utilizados no estudo 24 eram fêmeas e 20 machos de raças variadas. A idade dos cães variou entre um a nove anos (3,5 $\pm 2,07$ anos).

Para os hemogramas foram descartadas duas amostras, uma por estar coagulada e outra pela detecção de microfilária no esfregaço sanguíneo. Para as análises bioquímicas três amostras hemolisadas foram descartadas e a amostra do animal com microfilária.

Foram submetidos ao processo de validação um total de 25 parâmetros (13 hematológicos e 12 bioquímicos). Desse total, $76 \%(19 / 25)$ dos intervalos foram validados e $24 \%(6 / 25)$ foram rejeitados.

Para os parâmetros hematológicos o número de observações fora dos IRs propostos por Jain (1993) está demonstrado na tabela 1.
Tabela 1: Intervalos de referência (IRs) hematológicos estabelecidos por Jain (1993) para espécie canina e os resultados do procedimento de validação para cães domiciliados na Amazônia Oriental, Pará, Brasil com o total de amostras utilizadas $(n=20)$ e o total de amostras fora do intervalo de referência (n/20).

\begin{tabular}{lccc}
\hline \multicolumn{1}{c}{ Parâmetros } & $\begin{array}{c}\mathbf{1}^{\text {a }} \\
\text { amostragem }\end{array}$ & $\begin{array}{c}\mathbf{2}^{\text {a }} \\
\text { amostragem }\end{array}$ & IRs* $^{*}$ \\
\hline Hemácia $\left(\times 10^{6} / \mathrm{ul}\right)$ & $2 / 20$ & - & $5.5-8.5$ \\
Hemoglobina $(\mathrm{g} / \mathrm{dL})$ & $7 / 20$ & - & $12-18$ \\
Hematócrito $(\%)$ & $2 / 20$ & - & $37-55$ \\
VCM (fl) & $0 / 20$ & - & $60-77$ \\
CHGM (\%) & $3 / 20$ & $4 / 20$ & $32-36$ \\
Leucócitos $\left(\times 10^{3} / \mu \mathrm{L}\right)$ & $0 / 20$ & - & $6.0-17.0$ \\
Monócitos $\left(\times 10^{3} / \mu \mathrm{L}\right)$ & $2 / 20$ & - & $0.15-1.35$ \\
Linfócitos $\left(\times 10^{3} / \mu \mathrm{L}\right)$ & $3 / 20$ & $6 / 20$ & 1.0 \\
Neut. Segm. $\left(\times 10^{3} / \mu \mathrm{L}\right)$ & $1 / 20$ & - & $3.0-11.5$ \\
Neut. Bast. $\left(\times 10^{3} / \mu \mathrm{L}\right)$ & $0 / 20$ & - & $0-0.30$ \\
Eosinófilos $\left(\times 10^{3} / \mu \mathrm{L}\right)$ & $7 / 20$ & - & $0.1-1.25$ \\
Basófilos $\left(\times 10^{3} / \mu \mathrm{L}\right)$ & $0 / 20$ & - & Raros \\
Plaquetas $\left(\times 10^{3} / \mu \mathrm{L}\right)$ & $2 / 20$ & - & $200-500$ \\
\hline
\end{tabular}

Traços (-) indicam que as amostras não foram testadas na segunda amostragem. VCM - Volume Corpuscular Médio; CHGM - Concentração de Hemoglobina Corpuscular Média; *Jain (1993).

Com base nesses resultados, o método validou 69,2\% (9/13) dos parâmetros. Os resultados obtidos para eosinófilos e 
hemoglobina foram rejeitados na primeira amostragem, com sete amostras fora do intervalo de referência. Os resultados de linfócitos e CHGM tiveram que ser submetidos a uma segunda amostragem e permaneceram não validados. Os demais parâmetros hematológicos foram validados no primeiro procedimento de amostragem. Para os quatro parâmetros não validados (CHGM, hemoglobina, linfócitos e eosinófilos) foram estabelecidos novos IRs (Tabela 3).
Para bioquímica foram validados 83,3\% (10/12) dos parâmetros testados. Apenas dois (albumina e GGT) foram rejeitados na primeira amostragem, pois mais de cinco amostras estavam fora do intervalo de referência proposto por Kaneko (1997). Os resultados de proteína total e uréia tiveram que passar por uma segunda amostragem, pois três amostras ficaram fora do intervalo, e foram validados. Os demais parâmetros foram validados (tabela 2).

Tabela 2: Intervalos de referência (IRs) bioquímicos estabelecidos por Kaneko (1997) para espécie canina e o resultado do procedimento de validação para cães domiciliados na Amazônia Oriental, Pará, Brasil com o total de amostras utilizadas $(n=20)$ e o total fora do intervalo de referência $(n / 20)$.

\begin{tabular}{|c|c|c|c|}
\hline Parâmetros & $1^{\text {a }}$ amostragem & $2^{\mathrm{a}}$ amostragem & IRs* \\
\hline Albumina (g/L) & $9 / 20$ & - & $26-33$ \\
\hline ALT (U/L) & $0 / 20$ & - & $0-102$ \\
\hline AST (U/L) & $1 / 20$ & - & $0-66$ \\
\hline Colesterol (mg/dl) & $2 / 20$ & - & $135-275$ \\
\hline Creatinina (mg/dl) & $0 / 20$ & - & $0,5-1,5$ \\
\hline Fosfatase alcalina(U/L) & $2 / 20$ & - & $0-156$ \\
\hline GGT (U/L) & $7 / 20$ & - & $0-10$ \\
\hline Glicose (mg/dl) & $1 / 20$ & - & $65-118$ \\
\hline Globulinas (g/L) & $2 / 20$ & - & $27-44$ \\
\hline Proteínas totais (g/L) & $3 / 20$ & $0 / 20$ & $54-71$ \\
\hline Triglicerídeos (mg/dl) & $0 / 20$ & - & $20-112$ \\
\hline Uréia (mg/dl) & $3 / 20$ & $2 / 20$ & $21-60$ \\
\hline
\end{tabular}

Traços (-) indicam que as amostras não foram testadas na segunda amostragem. ALT - Alanina Aminotransferase; AST aspartato aminotransferase; GGT - Gama Glutamil Transferase. *Kaneko (1997).

Para albumina e GGT foram estabelecidos novos IRs que estão demonstrados na Tabela 3.

Tabela 3: Intervalos de referência (IRs) estabelecidos para os parâmetros hematológicos e bioquímicos não validados para cães domiciliados na Amazônia Oriental, Pará, Brasil.

\begin{tabular}{|c|c|c|c|c|c|c|c|c|c|c|c|}
\hline Parâmetros & $\mathbf{N}$ & Média & Mediana & DP & Min. & Max. & Outilers & $\begin{array}{c}\text { IR } \\
\text { (IC } 90 \%)\end{array}$ & $\begin{array}{c}\text { IR } \\
\text { (IC } 90 \%)\end{array}$ & Dist & Método \\
\hline Albumina (g/L) & 39 & 34 & 34 & 0,3 & 25 & 41 & 1 & $\begin{array}{c}26 \\
(24-29)\end{array}$ & $\begin{array}{c}41 \\
(39-42)\end{array}$ & NG & Rob \\
\hline GGT (U/L) & 40 & 10,6 & 11 & 2,7 & 5 & 16 & 1 & $\begin{array}{c}5 \\
(5,0-6,0)\end{array}$ & $\begin{array}{c}16 \\
(15,0-16,0)\end{array}$ & NG & NP \\
\hline Hemoglobina $(\mathrm{g} / \mathrm{dL})$ & 40 & 16,5 & 17 & 2,3 & 11,7 & 19,8 & 2 & $\begin{array}{c}11,7 \\
(11,7-12)\end{array}$ & $\begin{array}{c}19,8 \\
(19,3-19,8)\end{array}$ & NG & NP \\
\hline CHGM (\%) & 40 & 34,3 & 35,2 & 2,0 & 30 & 36 & 2 & $\begin{array}{c}30 \\
(30,2-30,5)\end{array}$ & $\begin{array}{c}36 \\
(36-36)\end{array}$ & NG & NP \\
\hline Linfócitos $\left(\times 10^{3} / \mu \mathrm{L}\right)$ & 39 & 3,81 & 3,70 & 1,39 & 1,44 & 7,37 & 3 & $\begin{array}{c}1,5 \\
(1,26-1,88)\end{array}$ & $\begin{array}{c}7,0 \\
(6,24-8,08)\end{array}$ & NG & Rob \\
\hline Eosinófilos $\left(x 10^{3} / \mu \mathrm{L}\right)$ & 41 & 1,14 & 0,99 & 0,84 & 0,15 & 3,69 & 1 & $\begin{array}{c}0,15 \\
(0,15-0,27)\end{array}$ & $\begin{array}{c}3,68 \\
(2,65-3,69)\end{array}$ & NG & NP \\
\hline
\end{tabular}

$\mathrm{N}=$ número de animais utilizados, $\mathrm{DP}=$ Desvio padrão, $\mathrm{Min} .=$ mínimo, $\mathrm{Max}=$ máximo, $\mathrm{IR}=$ intervalo de referência, $\mathrm{IC}=$ intervalo de confiança; Dist.= distribuição dos dados; NG= não gaussiana, Rob= método robusto; NP.= Não paramétrico. GGT - Gama Glutamil Transferase; CHGM - Concentração de Hemoglobina Corpuscular Média.

\section{Discussão}

Na prática veterinária o básico da interpretação dos exames laboratoriais é a comparação dos resultados do paciente com os IRs estabelecidos para a espécie. Dessa forma, os intervalos utilizados no laboratório devem ser confiáveis e representar a população atendida (FRIEDRICHS, 2010). Apesar disso, a grande maioria dos laboratórios se preocupa apenas em empregar métodos de controle de qualidade da parte analítica e não dão a devida importância à validade dos IRs utilizados nos seus laudos (TATE et al., 2015). 
Para cães os IRs estabelecidos por Jain (1993) e Kaneko (1997), são frequentemente utilizados nos laudos de exames. Esses intervalos foram estabelecidos há mais de 20 anos e podem não ser mais representativos da atual população canina. Assim, pesquisadores recomendam que cada laboratório estabeleça seus próprios IRs ou valide os que já estão disponíveis (FRIEDRICHS, 2010, TATE et al., 2015).

O presente estudo utilizou o procedimento de validação proposto pelo CLSI e encontrou que, para a maioria dos resultados hematológicos e bioquímicos, os intervalos de Jain (1993) e Kaneko (1997) foram validados. A metodologia de análise dos dados adotada para os parâmetros não validados também foi utilizada por Chung et al. (2016) para estabelecer o IRs com um $\mathrm{N}$ amostral de 38 cães de raças variadas, enquanto Nielsen et al. (2010) utilizaram 32 cães Bernesse da Montanha.

Nas análises hematológicas o intervalo estabelecido para hemoglobina (11,7 a 19,8 g/dL) foi superior ao limite máximo estabelecido por Jain (1993) (12 a $18 \mathrm{~g} / \mathrm{dL}$ ). Na veterinária a interpretação da concentração de hemoglobina é semelhante a do hematócrito e, pelo fato de ser grosseiramente equivalente a este, não tem muita utilidade para interpretações clínicas.

A maioria dos veterinários está mais familiarizada ou tem mais experiência em interpretar o hematócrito. Apesar dos nossos valores para $\mathrm{Hb}$ terem apresentado valores superiores ao limite máximo preconizados por Jain (1993) eles estão semelhantes aos valores de outros estudos que também encontraram resultados de hemoglobina mais elevados. Abella et al. (2011) estabeleceram o intervalo de 12,4 a 19,2 g/dl para cães de diferentes raças. Já Campora et al. (2011) encontraram valores superiores aos do nosso trabalho $(16,9$ a $22,8 \mathrm{~g} / \mathrm{dL})$ para cães da raça galgo, assim como Lavoué et al. (2014) (12,8 a 20,6 g/ dL) para cães da raça dogue de bordéus.

Para a CHGM os valores foram inferiores ao limite mínimo (30 a $36 \%$ ) quando comparados com os IRs de Jain (1993) (32 a $36 \%$ ). Entretanto, apesar dos valores estarem inferiores, esses são similares a outros estudos. Moritz et al. (2004) obtiveram intervalo de 31,6 a $34,3 \%$ e Serra et al. (2012) também observaram essa diminuição $(30,8$ a $35,5 \%)$ ambos para cães de raças variadas, corroborando com o presente estudo.

Neste estudo, todos os animais estavam com histórico de vermifugação em dia e sem relatos de ocorrência de doenças alérgicas, apesar disso os valores de eosinófilos $(0,15$ a 3,68 x $10^{3} / \mu \mathrm{L}$ ) foram extrapolaram os limites mínimo e máximo

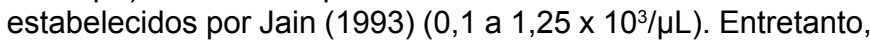
há relatos de valores superiores aos definidos por Jain (1993), a exemplo dos estudos de Abella et al. (2011) $(0,1$ a 3,1 x 103/ $\mu \mathrm{L})$ em cães de raças e dos achado de Lavoué et al. (2014) $(0,3$ a 3,3 $\times 10^{3} / \mu \mathrm{L}$ ) em cães da raça dogue de bordeaux.

No entanto, um processo de hipersensibilidade subclínica não pode ser totalmente descartado, pois $50 \%$ dos animais utilizados no presente estudo eram da raça Golden Retriever. Há estudos que relatam a tendência dos animais dessa raça animais apresentarem dermatite atópica canina (DAC), podendo estar a eosinofilia associada a suas características fisiológicas influenciadas pelo padrão racial (ZUR et al., 2002, HARVEY et. al., 2019).

Embora o número de linfócitos do nosso estudo tenha sido superior aos limites mínimo e máximo de Jain (1993), ele está de acordo com Abella et al. (2011) que também encontrou valores mais elevados ( 1,1 a $\left.5,3 \times 10^{3} / \mu \mathrm{L}\right)$ que a referência padrão. Uma das possíveis causas dessa diferença no número de linfócitos seria a maior frequência de vacinações que os cães recebem atualmente, já que vários autores associam as imunizações como um fator importante para linfocitose (BUSH, 1992; MEYER et al., 1992; STRASSER et al., 2003). Embora menos provável, não se pode descartar que o aumento dos linfócitos possa ter ocorrido como alteração pré-analítica causada por estresse no momento da coleta. Segundo Thrall et al. (2015), o aumento no número de linfócitos pode ocorrer em resposta à excitação, podendo ser observada contagens de até $12 \times 10^{3} / \mu \mathrm{L}$, estando esta condição normalmente acompanhada de neutrofilia (JAIN, 1993).

Nas determinações bioquímicas, o intervalo de referência obtido para albumina $(26$ a $41 \mathrm{~g} / \mathrm{L}$ ) foi superior ao limite máximo estabelecido por Kaneko (1997) (26 a $33 \mathrm{~g} / \mathrm{L}$ ). Em trabalhos com a espécie canina foram determinados IRs semelhantes ao obtido no presente estudo. Em Bernese da Montanha, os valores de albumina obtidos foram de 26 a $44 \mathrm{~g} / \mathrm{L}$ (NIELSEN et al., 2010). Lavoué et al. (2013) encontraram o intervalo de 27 a $38 \mathrm{~g} / \mathrm{L}$ para albumina em cães da raça Dogue de Bordeaux. Chung et al. (2016), ao utilizarem cães de raças variadas, obtiveram um intervalo de 30 a $44 \mathrm{~g} / \mathrm{L}$.

Visando a manutenção de uma produção normal de albumina é necessário que o estado nutricional esteja adequado, e a ingestão de proteínas é de fundamental importância (COOPER et al., 2009). Atualmente, a maior parte dos cães domiciliados é submetida ao manejo alimentar de cães é baseado na utilização de rações comerciais que são formuladas para atender todas as necessidades nutricionais dos animais. Entretanto, a alimentação de cães utilizadas no presente estudo era baseada em alimentação caseira e a ingestão de proteínas poderia não ser a quantidade ideal e isso pode explicar as diferenças observadas. González et al. (2001) encontraram menores valores de albumina em cães e atribuíram esse achado ao tipo de alimentação dos animais que era baseado em alimentação caseira. Segundo Chung et al. (2016), o manejo alimentar de cães pode influenciar nos resultados do IRs obtidos.

Para os resultados da atividade de GGT, observou-se um intervalo (5,0 a 16,0 U/L) superior aos limites mínimo e máximo previamente estabelecido (0 a $10 \mathrm{U} / \mathrm{L}$ ) por Kaneko (1997). Outros autores encontraram valores semelhantes aos obtidos no presente estudo. Lavoué et al. (2013) obtiveram o intervalo de $<5$ a 12,4 U/L, Nielsen et al. (2010) estabeleceu o intervalo de 0 a 12,2 U/L e Chung et al. (2016) também encontraram que a atividade da GGT (3 a $11 \mathrm{U} / \mathrm{L}$ ) foi superior quando comparada com os IRs previamente estabelecidos. A elevação na atividade da GGT é geralmente atribuída a colestase, hepatopatias e indução por medicamentos (CHAPMAN e HOSTUTLER, 2013). Os animais selecionados não estavam fazendo uso de medicações que pudessem influenciar na atividade dessa enzima. Além disso, outros parâmetros que indicam a possibilidade de doença hepatobiliar (AST, ALT e FA) estavam dentro dos IRs preconizados por Kaneko (1997).

Em relação aos resultados das análises hematológicas e bioquímicas, as diferenças observadas entre os intervalos atuais e o proposto por Jain (1993) e Kaneko (1997) podem ser explicadas também pela variabilidade induzida pelo uso de diferentes equipamentos utilizados nas análises, critérios 
de amostragem, fatores pré-analíticos, além de questões demográficas e genéticas relativas aos animais (NIELSEN et al., 2010; CAMPORA et al., 2011). Estudos já compararam o desempenho em diferentes analisadores e obtiveram resultados desiguais para as mesmas analises (MORITZ et al., 2004; FLATLAND et al., 2014). Além disso, variações biológicas intrínsecas de cada indivíduo põem causar oscilações fisiológicas ao longo do tempo e contribuir para variações nos parâmetros analisados. (WALTON, 2012; BRAGA e PANTEGHINI, 2016). Já existem pesquisas que relatam a variação biológica em valores bioquímicos de cães e gatos (BARAL et al., 2014; RUAUX et al., 2012).

\section{Referências}

ABELLA, B.N.; GEFFRÉ, A.; CONCORDET, D.; BRAUN, J.P.; TRUMEL, C. 2011. Canine RI for the sysmex XT-2000iV hematology analyser. Veterinary Clinical Pathology, v. 40, n.3, p. 303-305, 2011.

BARAL, R.M.; DHAND, N.K.; FREEMAN, K.P.; KROCKENBERGER, M.B.; GOVENDIR, M. Biological variation and reference change values of feline plasma biochemistry analytes. Journal of Feline Medicine and Surgery, v. 16, p. 317325, 2014.

BIRGEL JÚNIOR, E.H.; D'ANGELINO, J.L.; BENESI, F.J.; BIRGEL, E.H. Valores de referência do leucograma de bovinos da raça Jersey criados no Estado de São Paulo. Brazilian Journal of Veterinary Research and Animal Science, v. 38, p. 136-141, 2001.

BRAGA, F.; PANTEGHINI, M. Generation of data on within-subject biological variation in laboratory medicine: an update. Critical Reviews in Clinical Laboratory Sciences, v. 53, p. 313-325, 2016.

BUSH, B.M. Interpretation of laboratory results for small animal clinicians. Oxford: Blackwell Scientific Publications, 1992, 515 p.

CAMPORA, C.; FREEMAN, K.P.; LEWIS, F.I.; GIBSON, G.; SACCHINI, F.; VAZQUEZ, M.J.S. Determination of haematological reference intervals in healthy adult greyhounds. Journal of Small Animal Practice, v. 52, p. 301-309, 2011.

CHUNG, S.H.; CHANG, L.W.; CHENG, T.L.; LIN, C.J.; WENYING CHEN, W.Y.; CHOU, C.C. Establishing in-house reference intervals for dogs in veterinary clinics. Taiwan Veterinary Journal, v. 42, n. 2, p. 53-67, 2016.

CHAPMAN, S.E.; HOSTUTLER, R.A. A Laboratory Diagnostic Approach to Hepatobiliary Disease in Small Animals. Veterinary Clinics: Small Animal Practice, v. 43, p. 1209-1225, 2013.

CONCEA. RN no 22, de 25 de junho de 2015: Baixa o Capítulo "Estudos conduzidos com animais domésticos mantidos fora de instalações de instituições de ensino ou pesquisa científica" do Guia Brasileiro de Produção, Manutenção ou Utilização de Animais em Atividades de Ensino ou Pesquisa Científica do Conselho Nacional de Controle e Experimentação Animal CONCEA. Disponível em: <https://www.in.gov.br/materia/-/asset publisher/Kujrw0TZC2Mb/content/id/33253223/do1-2015-10-02resolucao-normativa-n-22-de-25-de-junho-de-2015--33253219> Acesso em 20 fev. 2021.

COOPER, S.E.; WELLMAN, L.M.; CARSILLO, E.M. Hyperalbuminemia associated with hepatocellular carcinoma in a dog. Veterinary Clinical Pathology, v. 38, n. 4, p. 516-520, 2009.

DAVOINE, F.; LACY, P. Eosinophil cytokines, chemokines, and growth factors: emerging roles in immunity. Frontiers in Immunology, v. 5, p. 1-17, 2014.

\section{Conclusão}

Para a maioria dos parâmetros hematológicos e bioquímicos os IRs estabelecidos previamente na literatura ainda são válidos para utilização em cães criados na Amazônia Oriental. Para os parâmetros não validados, apenas para eosinófilos e linfócitos recomendamos que outros trabalhos, com um número maior de animais, sejam realizados para confirmar nossos achados ou estabelecer novos IRs. Os IRs estabelecidos para hemoglobina, CHGM, albumina e GGT podem ser utilizados em substituição aos intervalos antigos.

Conflitos de Interesse: Não há conflitos de interesse.

FERREIRA, C.E.S.F.; ANDRIOLO, A. Intervalo de referencia no laboratório clínico. Jornal Brasileiro de Patologia e Medicina Laboratorial, v. 44, p. 11-16, 2008.

FLATLAND, B.; BREICKNER, L.C.; FRY, M.M. Analytical performance of a dry chemistry analyzer designed for in clinic use. Veterinary Clinical Pathology v. 43, p. 206-217, 2014.

FRIEDRICHS, K.R. Reference intervals: an essential, expanding, and occasionally equivocal standard. Veterinary Clinical Pathology v. 39, n. 2, p. 131-132, 2010.

FRIEDRICHS, K.R.; HARR, K.E.; FREEMAN, K.P.; SZLADOVITS, B.; WALTON, R.M.; BARNHART, K.F.; BLANCO-CHAVEZ, J. ASVCP reference interval guidelines: determination of de novo reference intervals in veterinary species and other related topics. Veterinary Clinical Pathology, v. 41, p. 441-453, 2012.

GEORGE, J.W.; SNIPES, J.; LANE, V.M. Comparison of bovine hematology reference intervals from 1957 to 2006. Veterinary Clinical Pathology, v. 39, p. 138-148, 2010.

GEFFRÉ, A.; CONCORDET, D.; BRAUN, J.P.; TRUMEL, C. Reference Value Advisor: a new freeware set of macroinstructions to calculate reference intervals with Microsoft Excel. Veterinary Clinical Pathology, v. 40, n. 1, p. 107-112, 2011.

GONZÁLEZ, F.H.D.; CARVALHO, V.; MÖLLER, V.A.; DUARTE, F.R. Perfil Bioquímico Sanguíneo de Cães e Gatos na Cidade de Porto Alegre, Rio Grande Do Sul, Brasil. Arquivos da Faculdade de Veterinária. UFRGS, v. 29, n. 1, p. 1-6, 2001.

HARVEY, N.D.; SHAW, S.C.; CRAIGON, P.J.; BLOTT, S.C.; ENGLAND, G.C.W. Environmental risk factors for canine atopic dermatitis: A retrospective large-scale study in Labrador and golden retrievers. Veterinary Dermatology, v. 30, p. 396-119, 2019.

JAIN, N.C. Essentials of veterinary hematology. Philadelphia: Lea \& Febiger, 1993, $417 \mathrm{p}$.

KANEKO, J.J., HARVEY, J.W., \& BRUSS, M.L.1997. Clinical Biochemistry of Domestic Animals. San Diego: Academic Press, 1997, $932 \mathrm{p}$.

LAVOUÉ, R.; GEFFRÉ, A.; BRAUN, J.P.; PEETERS, D.; TRUMEL, C. Breed-specific biochemical reference intervals for the adult Dogue de Bordeaux. Veterinary Clinical Pathology, v. 42, n. 3, p. 346-359, 2013.

LAVOUÉ, R.; GEFFRE, A.; BRAUN, J.P.; PEETERS, D.; GRANAT, F.; BOURGES-ABELLA, N.; TRUMEL C. Breed-specific hematologic reference intervals in healthy adultm Dogues de Bordeaux. Veterinary Clinical Pathology, v. 43, n. 3, p. 352-361, 2014.

MEYER, D.J.; COLES, E.H.; RICH, L.J. Veterinary Laboratory Medicine: interpretation and diagnosis. Philadelphia: Saunders Company, 1992, 350p. 
MORITZ, A.; FICKENSCHER, Y.; MEYER, K.; FAILING, K.; WEISS, D.J. Canine and feline hematology reference values for the ADVIA 120 hematology system. Veterinary Clinical Pathology, v. 33, n. 1, p. 32-38, 2004.

NIELSEN, L.; HANSEN, M.K.; JENSEN, A.L.; KRISTENSE, A.T. Breed-specific variation of hematologic and biochemical analytes in healthy adult Bernese Mountain dogs. Veterinary Clinical Pathology, v. 39, n. 1, p. 20-28, 2010.

RAHMAN, M.K.; ISLAM, S.; FERDOUS, J.; UDDIN, M.H.; HOSSAIN, M.B.; HASSAN, M.M.; ISLAM, A. Determination of hematological and serum biochemical reference values for indigenous sheep (Ovis aries) in Dhaka and Chittagong Districts of Bangladesh. Veterinary World, v. 11, n. 8, p. 1089-1093, 2018

RUAUX, C.; CARNEY, P.C.; SUCHODOLSKI, J.S.; STEINER, J.M. Estimates of biological variation in routinely measured biochemical analytes in clinically healthy dogs. Veterinary Clinical Pathology, v. 41, p. 541-547, 2012.

RUGGERONE, B.; GIRALDI, M.; PALTRINIERI, S.; SCARPA, P. Hematologic and biochemical reference intervals in Shetland Sheepdogs. Veterinary Clinical Pathology, v. 47, p. 617-624, 2018.

RUSSELL, K.A.; ROUSSEL, A.J. Evaluation of the ruminal serum chemistry profile. Veterinary Clinics. Food Animal. Practice, v. 23, p. 403-426, 2007.
SERRA, M.; FREEMAN, K.P.; CAMPORA, C.; SACCHINI, F. Establishment of Canine hematology reference intervals for the Sysmex XT-2000iV hematology analyzer using a blood donor database. Veterinary Clinical Pathology, v. 41, n. 2, p. 207-215, 2012.

STRASSER, A.; MAY B.; TELTSCHER, A.; WISTRELA, E.; NIEDERMULLER, $\mathrm{H}$. Immune modulation following immunization with polyvalent vaccines in dogs. Veterinary Immunology and Immunopathology, v. 94, p. 113-121, 2003.

TATE, J.T., YEN, T.; JONES, R.D.G. Transference and Validation of Reference Intervals. Clinical Chemistry, v. 61, n. 8, p. 10121015, 2015.

THRALL, M.A.; WEISER, G.; ALISSON, R.W.; CAMPBELL, W.T. Hematologia e Bioquímica Clínica Veterinária. $2^{\mathrm{a}}$ ed. Guanabara Koogan, Rio de Janeiro, 2015.

WALTON, R.M. Subject-based reference values: biological variation, individuality, and reference change values. Veterinary Clinical Pathology, v. 41, p. 175-181, 2012.

ZUR, G.; IHRKE, P.J.; WHITE, S.D.; KASS, P.H. Canine atopic dermatitis: a retrospective study of 266 cases examined at the University of California, Davis, 1992-1998. Part I. Clinical features and allergy testing results. Veterinary Dermatology, v. 13, p. 89-102, 2002. 